\title{
ERAS Protocol in Bariatric Surgery
}

\author{
Carlos de la Paz Estrada* \\ Department of Anesthesiology and Reanimation, Provincial Pediatric Hospital, Cuba
}

*Corresponding author: Carlos de la Paz Estrada, Department of Anesthesiology and Reanimation, Provincial Pediatric Hospital, Cuba.
Received Date: June 22, 2020

Published Date: July 08, 2020

\begin{abstract}
Summary
The obese patient and, in particular, the morbid patient, represent one of the greatest challenges for the anesthesiologist, not only due to the difficulties in managing the airway, but also due to all the comorbidities associated with the disease, and its consequent repercussion on organs and systems. This places the obese at a clear disadvantage with respect to the normal patient from all points of view: medical, due to the countless anatomophysiological alterations; Diagnosis, since any technique, however simple it may be, (radiography, ultrasound, venous access, obtaining non-invasive blood pressure, etc.) is difficult and can delay diagnosis and practice, due to the difficulty in transfers, early mobilizations, among others. This means that the anesthetic-surgical act must be meticulously planned to anticipate the appearance of possible complications. There are various treatments that can be used to avoid it, they cover behavioral, dietary and medical aspects, but they are often close to failure due to how demanding they can be, this being the moment in which the patient accesses the bariatric surgery.
\end{abstract}

\section{Introduction}

Obese patients represent a real challenge for the anesthesiologist both technically and intellectually. This group of people has been found to be closely associated with increased perioperative morbidity and mortality. As a result, the anesthesiologist must be prepared to treat obese patients in the operating room and intensive care unit, so they must understand their pathophysiology and specific complications associated with their condition to make our treatment more effective in this group of patients. Undoubtedly, patients who suffer from this disease due to its complications become ill and die more frequently [1].

Obesity has become an epidemic condition around the world and has been related to numerous associated diseases such as coronary artery disease (CAD), cerebrovascular disease, type 2 diabetes mellitus, high blood pressure, certain cancers and ventilatory disorders during the dream [2].

The morbidity and mortality of the obese patient will be directly proportional to the degree of overweight and the duration of obesity and will be aggravated by a series of pathophysiological modifications that begin to affect young generations more and more every day. In these modifications there is a key participation of adipose tissue, which has become one of the most complex and fascinating endocrine organs discovered in recent times.

Adipose tissue is affected by genetic and environmental influences that make it dysfunctional. Visceral adipose tissue predominates in obesity, characterized by morphological and functional changes that make them a source of cytokines, currently known as adipokines, which lead to a low-grade chronic inflammatory state. The repercussion of these alterations is reflected in insulin resistance, endothelial injury and finally atherogenesis, which lead to chronic metabolic and cardiovascular complications, among others [3].

\section{Development and Discussion}

\section{The application of AGES in the practice of bariatric surgery}

The ERAS protocol (Enhanced Recovery After Surgery, for its acronym: Accelerated Recovery After Surgery)It originates from 
the 90's, when two groups of researchers presented different proposals to improve the postoperative evolution of patients undergoing elective surgery, emphasizing the importance of the information that is provided to the patient and their participation as an integral part of postoperative recovery. Then, especially in Europe, at the Hvidovre University Hospital in Denmark, Kehlet $\mathrm{H}$ [4] proposes new and different strategies to make the management of evolution in the perioperative more efficient. Delaney CP, et al. [5] focused her research on postoperative improvement, specifically diet management and early mobilization. Consequently, multimodal rehabilitation programs (RHMM), also called FastTrack, emerged. Taking these principles as a platform, traditional measures in anesthetic / surgical practice such as prolonged preoperative fasting, mechanical colon preparation, and the use of a nasogastric tube for decompression are not recommended. On the other hand, it is shown that practices such as intravenous analgesia for pain control, especially with opioids, the delay in the start of feeding until the appearance of peristalsis evaluated in a subjective way, and bed rest are risk factors that favor the increase in days of hospital stay and care costs $[6,7]$.

Fast-Track or ERAS programs were developed as multimodal programs with the aim of mitigating the loss of functional capacity and improving recovery in the perioperative period. The goal is to reduce morbidity and significantly improve recovery by reducing surgical stress with optimal pain control, mobilization, and early diet. As a consequence, the stay and costs are reduced.

In 2001 the ERAS group was formed, made up of different surgical teams from five countries in northern Europe (Scotland, Sweden, Denmark, Norway, and Holland). This group made a consensus that they called the ERAS project, characterized by an MRI program for patients undergoing elective surgery. This protocol includes a combination of preoperative, intraoperative, and postoperative strategies based on scientific evidence that improving the recovery and functionality of patients after the surgical event minimizes the response to surgical stress [8-10].

The fundamental goal of said protocol is to reduce metabolic stress caused by surgical trauma and at the same time support the patient's early recovery. Of course, acting on factors involved in the biological response to aggression influences postoperative complications and decreases hospital stay and hospitalization costs [11-13].

Point out that the protocol is made up of different strategies which are classified according to the perioperative period; These therapeutic interventions are aimed at decreasing the post-surgical stress response to improve postoperative evolution and thus decrease hospital stay without increasing morbidity and mortality, even in patients with high comorbidity [14-16].

Morbid obesity is an important risk factor for surgery because the traumatic effects on the abdominal wall of traditional laparotomy are responsible for a high percentage of postoperative complications. Laparoscopic surgery has undergone significant development in recent years. This less aggressive and simpler postoperative surgical technique benefits this group of patients compared to open surgery. Some studies place the risk of infection about 10 times less with laparoscopic surgery [17-19].

The implementation of ERAS in bariatric surgery could have a significant impact on perioperative care, due to the inherent morbidity and mortality attributed to the disease per se, given that, if perioperative care is not adequate, the patient's postoperative status degrades exponentially $[20,21]$.

As described, the project aims to modify the physiological, neuroendocrine, and psychological response to major surgery, so that it favorably influences the result. In bariatric surgery, as with other procedures, some ERAS concepts may not be applicable, however, other concepts such as the role of blockages, the use of minimally invasive surgery, immunonutrition and adequate glucose control play a fundamental role in improving recovery after surgeries [22].

The anesthesiologist is responsible for three specific elements that affect the result after this surgery: the first is the control of stress reactions to surgery, the second is fluid therapy, and last but not least, analgesia. The recognition of the importance of these three components of the ERAS protocol has led to the description of the well-known "trimodal approach" for the optimization of results in surgery by anesthesiologists [23].

Related to limiting the response to surgical stress, central regional anesthesia reduces activation of the neuroendocrine system and thereby prevents immunosuppression during surgery. We must mention several studies already known for their contribution to the subject, such as the Rem J, et al. [24] study, where it is described that epidural anesthesia combined with general anesthesia prevents lymphopenia and reduces postoperative agranulocytosis by $40 \%$, and is also related to a lower elevation. of catecholamines and serum cortisol, which is attributed to a lower endocrine-metabolic response, proposes that this would avoid postoperative immunosuppression. Ciepichal J and Kubler A [25] studied the effect on the functions of circulating neutrophils several years ago, such as their phagocytic capacity in patients undergoing subarachnoid block compared to other groups under general inhalation anesthesia but found no alterations in this cell type. Tonnesen E [26] studied the activity of the NKs comparing subarachnoid block and different techniques of general anesthesia, finding that this activity decreased in the two groups of general anesthesia. Koltun WA, et al. [27] did not find an alteration of NK cytotoxic capacity in patients under epidural anesthesia, but in the general anesthesia group, a significant drop in NK activity and an increase in plasma levels of catecholamines and cortisol were demonstrated, concluding that the maintenance of function NK would be a beneficial effect of epidural anesthesia especially in cancer surgery. 
There is a set of data that shows that regional anesthesia, especially epidural and spinal, is safe and viable in obese patients [28]. However, it is technically more complex due to the physical difficulty of placing the catheters and their tendency to migrate out of the epidural space. The dosage of local anesthetics through these catheters must be prudent, due to the increase in the cephalic distribution of the drug and the blockage induced by the smaller size of the epidural space than in patients of normal weight. The degree of respiratory compromise suffered by obese patients is also greater than that produced in normal-weight individuals with a high regional block.

Few data indicate that epidural analgesic treatment improves overall results. Because the use of laparoscopic surgery is spreading to the detriment of open laparotomy, this fact is less and less important. In the morbidly obese person undergoing open laparotomy, the use of epidural catheters to control pain through thoracic epidural anesthesia is the one that provides the greatest benefits in reducing postoperative attenuation of vital capacity $[29,30]$.Postoperative pain management also encompasses patient controlled intravenous analgesia (PCA) in addition to thoracic epidural analgesia. There are no data that certify the superiority of one technique over the other, so in many cases the surgical approach used, whether open or laparoscopic, is what determines the choice.

In this procedure, the use of short-acting anesthetics is essential: epidural analgesia has shown that it directly attenuates the postoperative stress response, reduces pain, and consequently improves lung function, as there is no restriction during breathing due to pain and promotes the return of intestinal function by blocking sympathetic activity and consequently reducing ileus. However, adverse effects have been described. Blocking the sympathetic nervous system may promote hypotension due to vasodilation. An increase in urinary retention has also been observed. The use of short-acting anesthetics favors the immediate recovery of the patient after surgery.

It is widely known that the most suitable analgesia is that method that must allow the safe and early mobilization of patients with a prompt return to normal function. In this sense, standardization within all centers would be the optimal condition since it would have the benefits of monitoring the effects of the regimen used on the results and supporting a coherent approach in the treatment rooms of all patients in the same [31]. The techniques that meet these principles continue to be regional anesthesia or local infiltration of the wound or peripheral nerve block, all applicable to the obese patient. This should produce a change in the use of intravenous opioids.

In patients undergoing bariatric surgery, adequate immunonutrition, adequate analgesia (non-opioid alternatives for pain control and minimally invasive surgery, antithrombotic prophylaxis, normothermia, adequate hydro electrolytic balance, and awareness of the importance of suspending toxic habits should be considered [32]).

Adherence to ERAS in bariatric surgery can improve patient outcomes, accelerate functional recovery, and decrease length of hospital stay; the application of this project in the surgery of the obese patient in addition to the benefit directly to the patient improves productivity and institutional cost savings [21].

The nutritional and metabolic complications of bariatric surgery include protein and protein-calorie malnutrition. Patients sometimes experience excessive weight loss suffering from severe steatorrhea or diarrhea, hypoalbuminemia, marasmus, edema, and hyperphagia [22]. In cases of severe malnutrition, patients may need nutritional, enteral, or parenteral treatment. Sometimes a surgical review is necessary to correct excessive weight loss and hypoalbuminemia. Therefore, an anesthetic regimen should be considered that considers the decrease in the binding effects of drugs due to low albumin concentrations.

\section{Conclusion}

The anesthetic management of an obese patient is complicated when the anesthesiologist is not familiar with the associated anatomical, physiological, and pharmacological changes. To obesity, which impact on the pharmacokinetics and pharmacodynamics of the drugs used. The main problem arises when deciding which type of anesthetic technique will be most beneficial for this type of patient.

\section{Acknowledgement}

None.

\section{Conflict of Interest}

No conflict of interest.

\section{References}

1. Adams JP, Murphy PG (2000) Obesity in anesthesia and intensive care, endocrine and metabolic disorders in anaesthesia and intensive care. $\mathrm{Br}$ J Anaesth 85(1): 91-108.

2. Artham SM, Lavie CJ, Patel HM, Ventura HO (2008) Impact of obesity on the risk of heart failure and its prognosis. J Cardiometab Syndr 3(3): 155-161.

3. Carrillo Esper R, de la Paz Estrada C (2012) Anesthetic management of the obese patient. Bishop publishing house, Mexico, pp: 223-235.

4. Kehlet $H$ (1997) Multimodal approach to control postoperative pathophysiology and rehabilitation. Br J Anaesth 78(5): 606-617.

5. Delaney CP, Fazio VW, Senagore AJ, Robinson B, Halverson AL, et al. (2001) Fast track postoperative management protocol for patients with high co-morbidity undergoing complex abdominal and pelvic colorectal surgery. Br J Surg 88(11): 1533-1535.

6. Kehlet H (2005) Fast-Track colonic surgery: status and prospectives. Recent Results Cancer Res 165: 8-13.

7. Małczak P, Pisarska M, Wysocki M, Major P, Pędziwiatr M (2018) Letter to the Editor Concerning the Publication: "Meta-Analysis of Enhanced Recovery Protocols in Bariatric Surgery". J Gastrointest Surg 22(8): 1462-1463. 
8. Fearon $\mathrm{KCH}$, Ljungqvist $\mathrm{O}$, Von Meyenfeldt $\mathrm{M}$, Revhaung A, Dejong $\mathrm{CHC}$ et al. (2005) Enhanced recovery after surgery: a consensus review of clinical care for patients undergoing colonic resection. Clin Nutr 24(3): 466-477.

9. Kehlet H, Wilmore D (2005) Fast-Track surgery. Br J Surg 92(1): 3-4.

10. Kehlet H, Wilmore D (2002) Multimodal strategies to improve surgical outcomes. Am J Surg 183(6): 630-641.

11. Jonsson A, Lin E, Patel L, Patel AD, Stetler JL, et al. (2018) Barriers to Enhanced Recovery after Surgery after Laparoscopic Sleeve Gastrectomy. J Am Coll Surg 226(4): 605-613.

12. Muñoz JL, Ruiz Tovar J, Miranda E, Berrio DL, Moya P, et al. (2016) C-Reactive Protein and Procalcitonin as Early Markers of Septic Complications after Laparoscopic Sleeve Gastrectomy in Morbidly Obese Patients Within an Enhanced Recovery After Surgery Program. J Am Coll Surg 222(5): 831-837.

13. Karlsson A, Wendel K, Polits S, Gislason H, Hedenbro JL (2016) Preoperative Nutrition and Postoperative Discomfort in an ERAS Setting: A Randomized Study in Gastric Bypass Surgery. Obes Surg 26(4): 743748.

14. Bindal V, John S, Dudeja U (2015) Enhanced recovery protocols - time to trim and adopt a lean bariatric practice. Surg Obes Relat Dis 11(4): 819-820.

15. Mannaerts GH, Van Mil SR, Stepaniak PS, Dunkelgrün M, De Quelerij M, et al. (2016) Results of Implementing an Enhanced Recovery After Bariatric Surgery (ERABS) Protocol. Obes Surg 26(2): 303-312.

16. Stowers MD, Lemanu DP, Hill AG (2015) Health economics in Enhanced Recovery After Surgery programs. Can J Anaesth 62(2): 219-230.

17. Lemanu DP, Singh PP, Berridge K, Burr M, Birch C, et al. (2013) Randomized clinical trial of enhanced recovery versus standard care after laparoscopic sleeve gastrectomy. Br J Surg 100(4): 482-489.

18. Blanchet MC, Gignoux B, Matussière Y, Vulliez A, Lanz T, et al. (2017) Experience with an Enhanced Recovery After Surgery (ERAS) Program for Bariatric Surgery: Comparison of MGB and LSG in 374 Patients. Obes Surg 27(7): 1896-1900.

19. Major P, Wysocki M, Torbicz G, Gajewska N, Dudek A, et al. (2018) Risk Factors for Prolonged Length of Hospital Stay and Readmissions After Laparoscopic Sleeve Gastrectomy and Laparoscopic Roux-en-Y Gastric Bypass. Obes Surg 28(2): 323-332.

20. Ahmed OS, Rogers AC, Bolger JC, Mastrosimone A, Robb WB (2018) Meta-Analysis of Enhanced Recovery Protocols in Bariatric Surgery. J Gastrointest Surg 22(6): 964-972.
21. Alvarez A, Goudra BG, Singh PM (2017) Enhanced recovery after bariatric surgery. Curr Opin Anesthesiol 30(1): 133-139.

22. Małczak P, Pisarska M, Piotr M, Wysocki M, Budzyński A, et al. (2017) Enhanced Recovery after Bariatric Surgery: Systematic Review and Meta-Analysis. Obes Surg 27(1): 226-235.

23. Sinha A, Jayaraman L, Punhani D, Chowbey P (2017) Enhanced Recovery after Bariatric Surgery in the Severely Obese, Morbidly Obese, SuperMorbidly Obese and Super-Super Morbidly Obese Using Evidence-Based Clinical Pathways: a Comparative Study. Obes Surg 27(3): 560-568.

24. Rem J, Brandt MR, Kehlet H (2005) Prevention of postoperative lymphopeniaand granulocytosis by epidural analgesia. Lancet 1(8163): 283-284.

25. Ciepichal J, Kubler A (1998) Effect of general and regional anesthesia on some neutrophil functions. Arch Immunol Ther Exp (Warsz) 46(3): 183-192.

26. Tonnesen E, Huttel MS, Christensen NJ (1987) Natural killer cell activity in patients undergoing minor gynaecological surgery. Eur J Anesthesiol 4(2): 119-125.

27. Koltun WA, Bloomer MM, Tilberg AF, Seaton JF, Ilahi O, et al. (1996) Awake epidural anesthesia is associated with improved natural killer cell cytotoxicity and a reduced stress response. Am J Surg 171(1): 68-73.

28. De Baerdemaeker L, Margarson M (1996) Best anaesthetic drug strategy for morbidly obese patients. Curr Opin Anaesthesiol 29(1): 119-128.

29. Warren JA, Stoddard C, Hunter AL, Horton AJ, Atwood C, et al. (2017) Effect of Multimodal Analgesia on Opioid Use After Open Ventral Hernia Repair. J Gastrointest Surg 21(10): 1692-1699.

30. Bamgbade OA, Oluwole O, Khaw RR. (2017) Perioperative Analgesia for Fast-Track Laparoscopic Bariatric Surgery. Obes Surg 27(7): 1828-1834

31. Singh PM, Panwar R, Borle A, Goudra B, Trikha A, et al. (2017) Efficiency and Safety Effects of Applying ERAS Protocols to Bariatric Surgery: a Systematic Review with Meta-Analysis and Trial Sequential Analysis of Evidence. Obes Surg 27(2): 489-501.

32. Proczko M, Kaska L, Twardowski P, Stepaniak P (2016) Implementing enhanced recovery after bariatric surgery protocol: a retrospective study. J Anesth 30(1): 170-173. 